\title{
MMW-HOTLINE
}

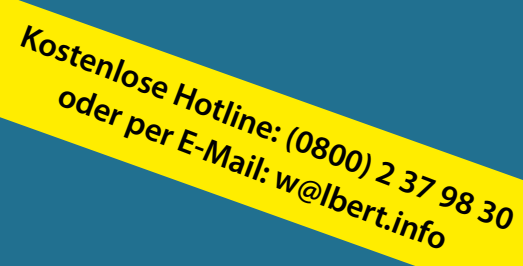

Leser der MMW können sich mit allen Fragen zur Abrechnung und Praxisführung an Helmut Walbert, Facharzt für Allgemeinmedizin, Würzburg, wenden. Sie erreichen ihn jeden Donnerstag von 13 bis 15 Uhr unter der kostenlosen Rufnummer (0800) 2379830 oder per E-Mail: w@lbert.info.

Helmut Walbert

Allgemeinarzt, Medizinjournalist und Betriebswirt Medizin

\section{Kann ich mir das Geld von den Kassen und den Patienten zurückholen?}

Frage von Dr. med. W., Allgemeinarzt: Mir ist aufgefallen, dass in meiner Praxis die Kosten für Porto und Telefon ständig steigen. Gibt es Möglichkeiten, Patienten oder Kostenträger an diesen Ausgaben zu beteiligen?

Antwort: Im Bereich der GKV gibt es das Kapitel 40.4 „Kostenpauschalen für die Versendung bzw. den Transport von Briefen, Szintigrammen und/oder schriftlichen Unterlagen, Kostenpauschale für Telefax": GOP 40120 bis 40 126. Diese Kostenpauschalen sind nach Briefgröße und -gewicht unterteilt. Sie kommen für den Versand von Patientenunterlagen und Arztbriefen an Mitbehandler in Frage.

Zu beachten ist, dass beim Versand von Untersuchungsmaterial entsprechende Verpackungen (Gefäße, Versandtüten, etc.) von den Untersuchern (Labor, Mikrobiologen, Pathologen usw.) anzufordern sind. Die Untersucher können eine Kostenpauschale einschließlich Porto in Ansatz bringen. Damit wird für die einsendende Praxis der Versand kostenneutral.

Anfragen der Krankenkassen sollten mit einem Freikuvert versehen sein. Ist dies nicht der Fall, kann auch in diesen Fällen eine entsprechende Portopauschale angesetzt werden. In diesem Zusammenhang sei auf die GOP 40144 „Kostenpauschale für fotokopierte oder EDV-technisch reproduzierte Befundmitteilungen, Berichte, Arztbriefe und andere patientenbezogene Unterlagen ausschließlich für den mit- oder

weiterbehandelnden oder konsiliarisch tätigen Arzt oder den Arzt des Krankenhauses", je Seite $€ 0,13$ hingewiesen. Diese GOP und die entsprechende Portopauschale kann - je nach regionaler KV-Regelung (bitte nachfragen!) - auch für Kopien verrechnet werden, wenn der die Kopie anfertigende Arzt für das Quartal, in dem dies erfolgt, über keinen Behandlungsausweis verfügt. Entweder er muss einen solchen vom derzeitigen Behandler anfordern oder - soweit dies von der regionalen Kassenärztlichen Vereinigung akzeptiert wird - sich selbst einen Überweisungsschein ausstellen und darf darauf diese Kosten abrechnen. Administrative oder organisatorisch in der Praxis

\section{Eintrag in ein Ärzteverzeichnis}

\section{Euro - für nichts!}

Frage von Dr. med. G. P., Würzburg: Ich erhalte per Fax immer wieder Angebote von Ärzteverzeichnissen, mich dort zu präsentieren. Zuletzt war es ein so genanntes „Zentrales Ärzteverzeichnis Bayern“. Was ist davon zu halten?

Antwort: Ab in den Papierkorb! Um in Zukunft nicht noch weitere Faxe zu erhalten: Schicken Sie ein Rückfax mit dem Hinweis, in Zukunft weitere Belästigungen zu unterlassen. entstehende Portokosten sind Allgemeinkosten der Praxis und sollten auf ihre Sinnhaftigkeit überprüft werden. Hier schlägt das Marketing mit dem Versand von „Arztbriefen in Kopie" an den Patienten und der kostenlosen Bereitstellung von Befundkopien für den Patienten in manchen Praxen kostenmäßig nicht unerheblich zu Buche. In der GOÄ ist für die Erstattung von Fotokopien und Faxbriefen die Berechnung der tatsächlich entstandenen Kosten der § 10 Abs. 1 GOÄ anzuwenden. Für E-Mails allerdings sind tatsächliche Kosten schwer berechen- und nachweisbar. Faxkosten können nur hilfsweise analog angesetzt werden.
Diese nutzlosen Verzeichnisse sind reine Abzocke. Im zitierten Fall wird mit Unterschrift ein Vertrag über mindestens zwei Jahre abgeschlossen mit "Un"-Kosten in Höhe von 1771 Euro, jährlich im Voraus zahlbar. Informationsgehalt des Verzeichnisses: gleich Null Es gibt genügend kostenlose Ärzteverzeichnisse - einschließlich der offiziellen Verzeichnisse der Kassenärztlichen Vereinigungen - mit weitaus höherem Informationswert. 